\title{
Factors Influencing Treatment Outcomes in Mandibular Class II Furcation Defects
}

\author{
Yi-Pin Tsao, * Rodrigo Neiva, ${ }^{*}$ Khalaf Al-Shammari, ${ }^{\dagger}$ Tae-Ju Oh, ${ }^{*}$ and Hom-Lay Wang*
}

Background: Factors influencing treatment outcomes in furcation defects remain to be studied. Therefore, the aim of the study was to evaluate the association between factors and clinical parameters that may influence treatment outcomes in mandibular Class II furcation defects.

Methods: Twenty-seven systemically healthy subjects with a Class II buccal or lingual furcation defect in lower molars were treated. Clinical measurements (probing depth [PD], clinical attachment level [CAL], recession, mobility, plaque index [PI], and bleeding on probing [BOP]) and defect (vertical and horizontal defect depths) were obtained at initial and 6-month reentry surgeries. Treatment modalities (e.g., nine each in the following three groups: open flap debridement [OFD] alone, bone graft [BG], and bone graft plus a bioabsorbable collagen membrane $[\mathrm{BG}+\mathrm{C}]$ ), anatomic factors (presence of cervical enamel projection, presence of cervical restorations/fixed prosthesis, and location of furcations [buccal or lingual sides; first or second molars]), clinical parameters (initial mobility, initial PD at furcation, initial CAL at furcation, mean initial PD of the tooth, mean initial CAL of the tooth, initial horizontal PD at furcation, initial horizontal/vertical defect depth, PI, and BOP), and background factors (endodontic status, smoking status, and surgeon's experience) were analyzed for associations with probability of clinical improvement.

Results: The anatomic factors, clinical parameters, and background information were found to have no effect in influencing treatment outcome with the exception of initial vertical defect depth. An initial vertical defect depth $\geq 4 \mathrm{~mm}$ had a borderline significance $(P=0.06)$ of achieving a high probability of having a $1-\mathrm{mm}$ vertical bone fill. In addition, treatment modality was found to be a major influence factor. Sites treated with BG were $16 \times$ more likely to have $>50 \%$ vertical bone fill than open flap surgery. Furthermore, sites treated with BG were $64 \times$ more likely to have a $1-\mathrm{mm}$ vertical defect fill compared to sites treated with OFD and $16 \times$ more likely to have a $2-\mathrm{mm}$ vertical defect fill compared to sites treated with OFD or BG + C. However, the additional membrane does not enhance the regenerative outcomes achieved by BG alone.

Conclusions: Initial vertical defect depth $(\geq 4 \mathrm{~mm})$ and treatment modality (e.g., BG) were found to be the clinical parameter and factor that were associated with high probability of clinical improvement. Sites treated with BG, such as mineralized human cancellous allograft, were more likely to have greater vertical furcation defect fill than the conventional OFD surgery. Additional membrane placement does not enhance the treatment outcome achieved by BG alone. J Periodontol 2006;77:641-646.

\section{KEY WORDS}

Allograft; bone; debridement; furcation defect; guided tissue regeneration; membranes.

\footnotetext{
* Department of Periodontics and Oral Medicine, University of Michigan School of Dentistry, Ann Arbor, MI

$\dagger$ Specialized Center for the Advancement of Dental Services, Ministry of Health, Kuwait, Kuwait.
}

$M$ olar teeth with furcation involvement are the most common teeth to be lost. ${ }^{1-4}$ Successful treatment to retain furcation-involved teeth remains one of the most difficult treatments in overall periodontal therapy. Methods for the treatment of furcation-involved molars have shown varying degrees of success. ${ }^{5-8}$ Factors contributing to this unpredictable and inconsistent outcome remain largely unclear. ${ }^{9}$

The etiologies of furcation involvement may include anatomic factors, extension of inflammatory periodontal disease, trauma from occlusion, pulpo-periodontal disease, and root fracture involving furcations. ${ }^{10} \mathrm{Fac}-$ tors that can affect the outcome of furcation therapy are complicated and difficult to control. ${ }^{9,11-14}$ Factors such as shallow initial probing depth (PD), poor oral hygiene, gingivitis, Actinobacillus actinomycetemcomitans infection, and absence of connective tissue cells on retrieved membranes have been shown to have less favorable results

doi: $10.1902 /$ jop.2006.050133 
for regenerative therapy. ${ }^{9-14}$ Cigarette smoking also exhibited a significant negative correlation to clinical outcome after guided tissue regeneration (GTR) treatment in controlled human clinical trials. ${ }^{13}$ Nonetheless, limited information is currently available with regard to how a variety of clinical factors may influence the predictability of clinical outcomes in furcation defect management. Therefore, the purpose of this study was to evaluate the association between factors and clinical parameters that may influence treatment outcomes in mandibular Class II furcation defects.

\section{MATERIALS AND METHODS}

\section{Study Design}

Twenty-seven systemically healthy subjects (12 females and 15 males; age range: 30 to 77 years), each with one mandibular Class II furcation defect ${ }^{5}$ (horizontal loss of periodontal support $\geq 3$ but $\leq 7 \mathrm{~mm}$ ) were enrolled in the study from the patient population of the University of Michigan School of Dentistry from January 13, 2003 through July 20, 2004. Presurgical treatments included oral hygiene instructions, scaling and root planing, and/or occlusal adjustment as indicated. Subjects were required to complete baseline examinations and were randomly assigned into three groups during the surgery after defect debridement and defect measurement. The random assignment was decided by drawing a piece of paper with either "A," "B," or "C," 10 each, from a brown bag. The three treatment methods were bone graft (BG; solventpreserved, mineralized human cancellous bone allograft $\left.{ }^{\ddagger}\right), B G$ plus collagen membrane§ $(B G+C)$, and open flap debridement (OFD) alone. All of the surgical procedures were either performed by or under the supervision of one clinician (YPT). The surgical procedure included the elevation of a full-thickness mucoperiosteal flap with intracrevicular incisions with two vertical releasing lines, preserving as much keratinized tissue as possible without reflecting the interdental papillae. The furcation defects were debrided, and all root surfaces were completely scaled and root planed by hand, ultrasonic, and rotating instruments." Cemento-enamel projection was removed by an enamel plastic burll if present. After debridement, patients were randomly assigned into one of the three groups: $B G$ alone (BG was mixed with normal saline and placed in the furcation area); $B G+C$ (after BG placement, collagen membrane was trimmed to cover the defect $2 \mathrm{~mm}$ beyond the edge of furcation defect); and OFD alone (all surgical procedures were identical except that no BG or $C$ were placed). The membrane was sutured with 5-0 chromic gut sutures ${ }^{\#}$ using the sling-suture technique. The flap was sutured securely to its original position with 5-0 polyglactin $910,{ }^{*}$ * with care to ensure that no BG or $C$ was left exposed. In OFD-treated sites, the furcations were covered by re- position of the flaps. None of the furcations were exposed after treatment. All patients received routine written and oral postoperative instructions and were instructed to rinse with $0.12 \%$ chlorhexidine gluconate for a period of 3 to 4 weeks. Sutures were removed 7 to 10 days after the surgery. Patients returned in 3 weeks after surgery for observation of any adverse tissue reactions. All patients received periodontal maintenance at 3 and 6 months.

A calibrated examiner ( $\mathrm{RN}$ ) recorded all clinical parameters (PD, clinical attachment level [CAL], bleeding on probing $[\mathrm{BOP}]$, plaque index [PI], and gingival index [GI]) at baseline and at 6-month postoperative visits. Direct defect measurement was performed using a prefabricated acrylic stent and a University of North Carolina (UNC) probe; the stent was grooved at the time of surgery to record the orientation of the probe. When the stent was placed on the occlusal surfaces of the treated tooth and adjacent dentition, an occlusal ledge served as an occlusal landmark. The open defect measurements included stent to the free gingival margin, stent to base of defect, crest of bone to base of defect, and bucco-lingual/mesio-distal morphology of the furcation defects. The bucco-lingual depth of the defect was measured from furcation entrance to the innermost part of the defect. The mesio-distal depth of the defect was measured at the crestal bone level. Vertical defect fill was calculated from the change of distances measured from the reference point on the stent to base of defect. Crestal bone resorption was also calculated from subtracting vertical bone fill from the change of defect depth. The examiner (RN) remained masked to the treatment types of all patients for the entire duration of the study.

The use of human subjects in this study was approved by the Health Science institutional review board of the University of Michigan. All subjects reviewed and signed the consent forms prior to entry in the study.

\section{Investigator Calibration}

Two sets of measurements were recorded at two separate occasions for each tooth. A 15-mm UNC probe and custom-made acrylic stent were used for measurements at both visits. The intraexaminer correlation or consistency was higher than $80 \%$ for this study. The examiner was instructed on the techniques for data collection and collected all measurements. The examiner (RN) was masked to the measurements recorded. All measurements were performed within the same day to avoid possible changes in the subjects (i.e., different levels of plaque control on different

\footnotetext{
‡ Puros, Zimmer Dental, Carlsbad, CA.

$\S$ BioMend Regular, Zimmer Dental.

Nemeyer's bur, Brasseler USA, Small Parts, Miami Lakes, FL.

I Brasseler USA, Small Parts.

\# Vicryl, Ethicon, Johnson \& Johnson, Somerville, NJ.

** Vicryl, Ethicon.
} 
days). Measurements were compared, and a minimal correlation of $80 \%$ was achieved. Calculations of intraexaminer consistency were performed before, during, and after research. A $\kappa$ value for the examiner (RN) was $88 \%$ before the project started, $89 \%$ during the project, and $91 \%$ after the project was completed.

\section{Statistical Analysis}

The data collected on continuous variables were presented as mean \pm SD for different groups and analyzed using a statistical software program. ${ }^{\dagger \dagger}$

The $\chi^{2}$ test and logistic regression analysis were used to assess the association of each of the following factors, including treatment modalities, anatomic factors (presence of cervical enamel projection [CEP], presence of cervical restorations/fixed prosthesis, location of furcations [buccal or lingual sides; first or second molars]), clinical parameters (initial mobility, initial PD at furcation, initial CAL at furcation, mean initial PD of the tooth, mean initial CAL of the tooth, initial horizontal PD at furcation, initial horizontal defect depth [HDD] and vertical defect depth [VDD], PI, and BOP), and background factors (endodontic status, smoking status, and surgeon's experience; and, e.g., a 1-, 2-, or 3-mm improvement in HDD; a 1-, 2-, or 3-mm improvement in VDD; improvement to a Class I furcation; and a 50\% improvement in VDD), and to estimate whether there is a significant increase in the odds of improvement associated with certain factors or clinical parameters. First, the $\chi^{2}$ test was performed individually for evaluation between each factor and binary indicator of clinical improvement. After estimates of effects on the clinical improvement of each potential predictor were derived, the logistic regression analysis was performed. Adjustments for multiple variances were performed in the model. For each defect on each patient, the clinical measurements were entered into the analyses. For each analysis, one main outcome variable was chosen (e.g., $50 \%$ vertical bone fill). To identify prognostic factors for therapeutic benefits, potential predictors were added to the final model according to a $P$ value $<0.05$. Smoking habits were defined by indicator variables. Statistical analysis was performed using statistic programs for general analysis and for multilevel analyses.

The Kruskal-Wallis test and analysis of variance (ANOVA) were used to compare the means of clinical parameter improvement over time (e.g., percentage of improvement in VDD and millimeters of improvement in VDD) between different treatment modalities. The paired $t$ test and repeated measures ANOVA were used to compare the mean difference of clinical measurements (e.g., HDD, VDD, CAL, and PD) among different treatment modalities over time (baseline and 6 months).

All tests of hypotheses were two sided, and differences were considered statistically significant when $P<0.05$ and with a 95\% level of confidence.

\section{RESULTS}

When stratifying the data by the anatomic factors (presence of CEP, presence of cervical restorations/ fixed prosthesis, and location of furcations), treatment modalities, clinical parameters (initial mobility, initial PD at furcation, initial CAL at furcation, mean initial PD of the tooth, mean initial CAL of the tooth, initial horizontal PD at furcation, initial horizontal and vertical defect depths, PI, and BOP), and background factors (endodontic status, smoking status, and surgeon's experience: divided into three groups in the order of the surgery that was performed), correlations between these factors and higher probability of achieving clinical improvement (e.g., 50\% vertical bone fill or a 1-, 2-, or 3-mm vertical/horizontal bone fill) were analyzed. There were no significant relationships indicating a higher probability of achieving clinical improvement among the presence of CEP, presence of cervical restorations/fixed prosthesis, initial mobility, location of furcations (buccal or lingual sides; first or second molars), initial mobility, initial PD at furcation, initial CAL at furcation, mean initial PD of the tooth, mean initial CAL of the tooth, initial horizontal PD at furcation, initial horizontal defect depth, initial PI at furcation, initial BOP at furcation, endodontic status, smoking status, and surgeon's experience. However, significant associations were revealed with the effect of treatment modality and initial vertical defect depth. The results from $\chi^{2}$ tests indicated a borderline significant $(P=0.06)$ association of initial vertical defect depth $\geq 4 \mathrm{~mm}$ and a higher probability of having $1-\mathrm{mm}$ vertical bone fill compared to an initial vertical defect depth $\leq 3 \mathrm{~mm}$. Significant evidence $(\beta=2.77 ; P=0.03$; odds ratio $=16)$ of an association was shown between treatment modality and vertical defect fill, with the BG group being $16 x$ more likely to have a $50 \%$ vertical defect fill compared to the OFD group and $64 \times(\beta=4.159 ; P=0.006$; odds ratio $=64)$ more likely to have a $1-\mathrm{mm}$ vertical defect fill compared to the OFD group (Table 1). An association $(\beta=2.773 ; P=0.003$; odds ratio $=16)$ between $B G+C$ and vertical bone fill was also identified; it indicated that the $\mathrm{BG}+\mathrm{C}$ group was $16 \times$ more likely to have a $1-\mathrm{mm}$ vertical defect fill compared to the OFD group. Furthermore, evidence $(\beta=2.773 ; P=0.03$; odds ratio $=16$ ) of an association between $B G$ and vertical bone fill, with the BG group being $16 \times$ more likely to have a 2 -mm vertical defect fill compared to the OFD and BG $+C$ groups.

When comparing the results among different treatment modalities, sites treated with BG showed the highest vertical bone fill followed by $B G+C$, whereas vertical bone loss was noted in sites treated with OFD (Table 2). Vertical bone fill in sites treated with BG and

$\dagger \dagger$ SPSS, Chicago, IL. 
Table I.

\section{Factors Associated With Higher Probability of Improvement}

\begin{tabular}{|c|c|c|c|c|c|}
\hline Outcome Variable & Variable & $\beta$ & Odds Ratio & 95\% Confidence Interval & $P$ Value \\
\hline $50 \%$ vertical defect fill & $\mathrm{T} \times(\mathrm{I})$ & 2.773 & 16.0 & $(13.5,18.5)$ & $0.030 *$ \\
\hline I-mm vertical defect fill & $\mathrm{T} \times(\mid)$ & 4.159 & 64.0 & $(61.1,66.9)$ & $0.006 *$ \\
\hline I-mm vertical defect fill & $\mathrm{T} \times(2)$ & 2.773 & 16.0 & $(13.5,18.5)$ & $0.030 *$ \\
\hline 2-mm vertical defect fill & $\mathrm{Tx}(\mathrm{I})$ & 2.773 & 16.0 & $(\mid 3.5,18.5)$ & $0.030 *$ \\
\hline 2-mm vertical defect fill & $\mathrm{T} \times(3)$ & 2.773 & 16.0 & $(13.5,18.5)$ & $0.030 *$ \\
\hline I-mm horizontal defect fill & $\mathrm{T} \times(\mathrm{I})$ & 1.946 & 7.0 & $(3.9,10.2)$ & 0.070 \\
\hline I-mm horizontal defect fill & $\mathrm{T} \times(2)$ & 1.946 & 7.0 & $(3.9,10.2)$ & 0.070 \\
\hline
\end{tabular}

Tx $(1)=$ BG compared to OFD; Tx (2) = BG + C compared to OFD; Tx (3) = BG compared to BG + C.

* Statistically significant $(P<0.05)$; $P$ values were based on logistic regression analysis.

Table 2.

\section{Results Obtained From Three Treatment Modalities}

\begin{tabular}{lc}
\hline Treatment & Mean \pm SD $(\mathrm{mm})$ \\
\hline Vertical bone fill* & $-1.6 \pm 2.1$ \\
OFD & $1.9 \pm 1.4^{\dagger}$ \\
BG & $0.7 \pm 0.9^{\dagger}$ \\
BG $+C$ & \\
Horizontal bone fill & $0.2 \pm 1.7$ \\
OFD & $1.1 \pm 0.9^{\dagger}$ \\
BG & $1.1 \pm 0.9^{\dagger}$ \\
BG $+C$ &
\end{tabular}

* Results of Kruskal-Wallis test. There is evidence of a significant difference of the mean of vertical bone fill between BG and OFD $(P=0.002)$ and between BG $+\mathrm{C}$ and OFD $(P=0.016)$

$\dagger$ Statistically significant $(P<0.05)$.

$\mathrm{BG}+\mathrm{C}$ was significantly higher than that in sites treated with OFD $(P<0.05)$. Horizontal bone fill was $0.2 \pm 1.7 \mathrm{~mm}, 1.1 \pm 0.9 \mathrm{~mm}$, and $1.1 \pm 0.9 \mathrm{~mm}$ for the OFD, BG, and BG + C groups, respectively. The reductions of horizontal defect depth and vertical bone fill were statistically significant in $B G$ and $B G+C$ groups $(P<0.05)$. However, horizontal bone fill, recession, CAL gain, and PD reduction at furcations showed no differences among different treatment modalities.

The mean differences of measurements over time among three groups were compared using the paired $t$ test and repeated measure ANOVA. When comparing the three treatment modalities in terms of the mean difference of vertical defect depth over time, there was evidence of a time effect $(P<0.05)$ and treatment effect $(P<0.05)$ for all groups. For the mean difference of horizontal defect depth, vertical probing depth at furcation, and horizontal probing depth, only time effect, and not treatment effect, was found.

\section{DISCUSSION}

Results obtained from this study indicated the positive effects of BG and BG + GTR barriers for the treatment of furcation defects, especially vertical defect fill. In addition, our data indicated that BG (e.g., human cancellous bone)-treated sites are $16 \times$ more likely to have $\geq 50 \%$ of vertical furcation defect fill than OFD.

Cigarette smoking is considered a behavioral and environmental factor related to the treatment outcome of subjects with furcation defects, and some negative correlations with regenerative outcomes have been reported. ${ }^{13,15-17}$ However, although 14 participants in the present study smoked $\geq 1$ pack/ day, and four subjects smoked $<1$ pack/day, smoking was found to have no effect on treatment outcome. A similar regenerative outcome was also reported in smokers compared to non-smokers in a recent study. ${ }^{9}$

There has been some controversy regarding the relationship between the pulpal status of teeth and their regenerative potential. Some have suggested that endodontically treated teeth may be less-than-ideal candidates for regenerative therapy. ${ }^{18}$ However, in agreement with Diem et al. ${ }^{19}$ and Chen et al., ${ }^{20}$ pulpal condition did not affect the treatment outcome in this study.

The exact nature of the tissue reorganization in the BG areas could not be ascertained in this clinical study. In a few cases, residual BG-like material was observed and was well incorporated with newly formed bone with hard surface texture. Histologic biopsy with a pretreatment landmark to show true regeneration would be important to verify the results obtained from this study. Future study in this area is recommended. 
Differences in root morphology and furcation access exist in mandibular first and second molars and in buccal and lingual furcations, which may affect surgical management. ${ }^{21,22}$ However, similar clinical improvement for first and second molars with Class II furcations after GTR therapy was reported..$^{9,12}$ In the present study, there was no significant correlation between the location of furcation defects (buccal or lingual; first or second molars) and the probability of improvement. Because of the limited sample size for comparison, the impact of the location of furcations on treatment outcomes cannot be concluded.

The presence of CEPs was identified in six (13.3\%) of 30 furcations. No correlation was found between the presence of CEPs and the probability of improvement of the clinical parameters. Cervical restorations and fixed prostheses were present on 14 (46.7\%) of the 30 teeth. No correlation was found between the presence of a cervical restoration or fixed prosthesis and the probability of improvement. Both CEPs and a prosthesis may contribute to the initiation of furcation involvement. ${ }^{23}$

The limitations of the regeneration in some subjects in the present study might relate to the proximity of the crown margins, proximal bone height, and location of the furcations. However, statistical results obtained from this study failed to show any correlation with a higher or lower probability of improvement in cases where furcations were located on lingual sides or in second molars. Likewise, the restorative or endodontic conditions of a tooth did not have any correlation with the changes of probability for clinical improvement. Physiological limitations are still present. The proximity of crown margins to furcations may inhibit the epithelial and/or connective tissue attachment to form coronally. Second molars and lingual furcations may cause access difficulties and, therefore, reduce the efficiency in debridement of the root surfaces and influence treatment outcome.

Initial vertical defect depth $(\geq 4 \mathrm{~mm})$ and treatment modality (e.g., BG) were found to be the clinical parameter and factor associated with a high probability of clinical improvement. Sites treated with BG, such as mineralized human cancellous allograft, were more likely to have a greater vertical furcation defect fill than sites treated by conventional OFD surgery. Additional membrane placement does not enhance the treatment outcome achieved by BG alone.

\section{ACKNOWLEDGMENTS}

This study was partially supported by the University of Michigan Periodontal Graduate Student Research Fund. The authors thank Dr. Paul Krebsbach, Department of Biologic and Materials Science, School of Dentistry, University of Michigan, and Brady West, Center for Statistical Consultation and Research, Uni- versity of Michigan, for their guidance and helpful comments. In addition, the authors thank Zimmer Dental, Carlsbad, California, for donating materials and providing a grant for the study.

\section{REFERENCES}

1. Ramfjord SP, Caffesse RG, Morrison EC, et al. 4 modalities of periodontal treatment compared over 5 years. J Clin Periodontol 1987;14:445-452.

2. Hirschfeld L, Wasserman B. A long-term survey of tooth loss in 600 treated periodontal patients. J Periodontol 1978;49:225-237.

3. Chace R Sr., Low SB. Survival characteristics of periodontally-involved teeth: A 40-year study. J Periodontol 1993;64:701-705.

4. McFall WT Jr. Tooth loss in 100 treated patients with periodontal disease: A long-term study. J Periodontol 1982;53:539-549.

5. Hamp SE, Nyman S, Lindhe J. Periodontal treatment of multirooted teeth: Results after 5 years. J Clin Periodontol 1975;2:126-135.

6. Hellden LB, Elliot A, Steffensen B, Steffensen JE. The prognosis of tunnel preparations in treatment of Class III furcations: A follow-up study. J Periodontol 1989;60: 182-187.

7. Carnevale G, Di Febo G, Tonelli MP, Marin C, Fuzzi M. A retrospective analysis of the periodontal-prosthetic treatment of molars with interradicular lesions. Int $J$ Periodontics Restorative Dent 1991;11:189-205.

8. Carnevale G, Pontoriero R, di Febo G. Long-term effects of root-resective therapy in furcation-involved molars: A 10-year longitudinal study. J Clin Periodontol 1998;25:209-214.

9. Bowers GM, Schallhorn RG, McClain PK, Morrison GM, Morgan R, Reynolds MA. Factors influencing the outcome of regenerative therapy in mandibular Class II furcations: Part I. J Periodontol 2003;74:1255-1268.

10. Newell DH. The diagnosis and treatment of molar furcation invasions. Dent Clin North Am 1998;42: 301-337.

11. Cortellini P, Pini-Prato G, Tonetti M. Periodontal regeneration of human infrabony defects (V): Effect of oral hygiene on long-term stability. J Clin Periodontol 1994;21:606-610.

12. Machtei EE, Cho MI, Dunford R, Norderyd J, Zambon JJ, Genco RJ. Clinical, microbiological, and histological factors which influence the success of regenerative periodontal therapy. $J$ Periodontol 1994;65: 154-161.

13. Trombelli L, Kim CK, Zimmerman GJ, Wikesjö UM. Retrospective analysis of factors related to clinical outcome of guided tissue regeneration procedures in intrabony defects. J Clin Periodontol 1997;24: 366-371.

14. Wang HL, MacNeil RL. Guided tissue regeneration: Absorbable barriers. Dent Clin North Am 1998;42: 505-522.

15. Tonetti MS, Pini-Prato G, Cortellini P. Effect of cigarette smoking on periodontal healing following GTR in infrabony defects: A preliminary retrospective study. $J$ Clin Periodontol 1995;22:229-234.

16. Luepke PG, Mellonig JT, Brunsvold MA. A clinical evaluation of a bioresorbable barrier with and without decalcified freeze-dried bone allograft in the treatment of molar furcations. J Clin Periodontol 1997;24: 440-446. 
17. Rosenberg ES, Cytryn F, Guzman A, Maldonado B. The role of guided tissue regeneration and guided bone regeneration. Ann $R$ Australas Coll Dent Surg 1994;12:80-88.

18. Sanders JJ, Sepe WW, Bowers GM, et al. Clinical evaluation of freeze-dried bone allografts in periodontal osseous defects: Part III. Composite freeze-dried bone allografts with and without autogenous bone grafts. J Periodontol 1983;54:1-8.

19. Diem CR, Bowers GM, Ferrigno PD, Fedi PF Jr. Regeneration of the attachment apparatus on pulpless teeth denuded of cementum in the rhesus monkey. $J$ Periodontol 1974;45:18-22.

20. Chen SY, Wang HL, Glickman GN, Shyr Y, MacNeil RL. Multivariate analysis of factors influencing outcomes of regenerative therapy: A retrospective study. Int Chin J Dent 2002;2(1):48-58.

21. Machtei EE, Dunford R, Grossi SG, Genco RJ. Gingival recession and exposure of barrier membrane: Effect on guided tissue regeneration of Class II furcation defects. Int J Periodontics Restorative Dent 1995;15: 590-599.

22. Mardam-Bey W, Majzoub Z, Kon S. Anatomic considerations in the etiology and management of maxillary and mandibular molars with furcation involvement. Int J Periodontics Restorative Dent 1991; 11:398-409.

23. Wang HL, Burgett FG, Shyr Y. The relationship between restoration and furcation involvement on molar teeth. J Periodontol 1993;64:302-305.

Correspondence: Dr. Hom-Lay Wang, Department of Periodontics and Oral Medicine, University of Michigan School of Dentistry, 1011 N. University Ave., Ann Arbor MI 48109-1078. Fax: 734/936-0374; e-mail: homlay@ umich.edu.

Accepted for publication October 7, 2005. 